\title{
Effects of Altered Dietary Fiber on the Gut Microbiota, Short-Chain Fatty Acids and Cecum of Chickens during Different Growth Periods
}

\author{
Baosheng Sun, Linyue Hou and Yu Yang * \\ Laboratory of Poultry Production, College of Animal Science and Veterinary Medicine, Shanxi Agricultural \\ University, Taigu 030801, China; xinxiaobao85@163.com (B.S.), sxauLynn@163.com (L.H.). \\ * Correspondence: sxauywd@126.com
}

\begin{abstract}
Increasing numbers of researchers are interested in the importance of dietary fiber for the gut microbiota, microbiotal metabolite SCFA, energy metabolism and gut health of the host. However, studies have demonstrated that long-term and longitudinal observation may be needed to evaluate the effect of dietary fiber better, and few such works have been made in chickens. Therefore, we successively fed low-fiber, high--fiber and low-fiber diets to two breeds of chickens during different growth periods (1-8, 9-20 and 21-50 weeks), aiming to longitudinally observe the long-term effect of altered dietary fiber on the gut microbiota, SCFA and development of cecum of chickens with age. The results showed that the composition and function of the gut microbiota, SCFA and the development of the cecum were different during different periods, which was largly affected by dietary fiber. However, the causes of some effects were different during the different periods. For example, compared with that in low-fiber chickens at 8 weeks, dominant fiber-degradation bacteria such as Bacteroidetes, Alloprevotella and SCFA-producing bacteria such as Faecalibacterium increased due to a high-fiber diet at 20 weeks, while due to a high feed intake in 50 weeks. Moreover, the concentration of SCFA in 20 weeks was significantly higher than in 8 weeks and 50 weeks, but the causes of this difference were also distinct. It was proposed that a long-term observation was needed to evaluate the effect of dietary fiber better on chickens. The metabolite pathways of ATP-binding cassette (ABC) transporters encoded by Firmicutes were enriched in 8 weeks, while a two-component system and $\beta$-glucosidase encoded by Bacteroidetes were enriched in 20 and 50 weeks. The trend was the same in two breeds of chickens except for Alloprevotella. In addition, the total content of SCFA in the contents of cecum was also affected by the size of the cecum. Surprisingly, the length of the cecum shortened from 20-50 weeks, maybe due to reduced dietary fiber.
\end{abstract}

Keywords: dietary fiber; SCFA; growth period; chickens; gut microbiota; microbiotal diversity; age; cecum

\section{Introduction}

Dietary fiber is the substrate of microorganisms residing in the gut. The consumption of a high-fiber diet helps to increase the richness and diversity of gut microbiota [1]. In contrast, a lack of dietary fiber will decrease microbial diversity $[2,3]$ and reduce the thickness of the mucus layer [4]. High microbiota diversity and varied complex carbohydrates such as dietary fiber in the diet might result in increased production of multiple types of short-chain fatty acids (SCFAs) [5]. It seems to be puzzling that the microbiotal diversity of obese people decreases [6,7], but their SCFAs were higher than lean people $[8,9]$. It is unclear whether SCFA contribute to obesity or simply reflect an altered gut microbiota [10]. The exact relationship between microbiotal diversity and SCFA is worth being 
explored. Dietary fiber is broken down into monosaccharide or oligosaccharide by carbohydrate active enzymes (CAZymes) such as $\beta$-glucosidase encoded by microbiotal genomes [11]. Bacteroidetes is the generalist of degrading dietary fiber [12] while Firmicutes is regarded as the specialists. Bacteroides and Prevotella are fiber-degradation experts in Bacteroidetes. Bacteroidetes can utilize a series of plant-derived dietary polysaccharides via unique polysaccharide utilization sites (PULs) [13]. In contrast, Firmicutes introduces glucose via ATP-binding cassette (ABC) transporters and other transporters encoded by gram-positive PULs (gpPULs) [14]. Excellent fiber-degradation bacteria in Firmicutes include Ruminococcus, Fibrobacter, Butyrivibrio and so on. Ruminococcus can efficiently utilize cellulose via cellulosome [15].

Some monosaccharides are used as a carbon source and for energy by microorganisms, while some of them are fermented into SCFAs by SCFA-producing bacteria. Bifidobacterium produces acetate using bifid-shunt [16]. Propionibacterium produces propionate via a succinate-propionate pathway [17]. Faecalibacterium and Roseburia can use acetate to generate butyrate by butyryl-CoA:acetyl-CoA transferase [18]. Acetate services a source of energy for peripheral tissues. Propionate synthesizes glycogen in the liver. Butyrate provides energy for normal colonic epithelial cells [19] and promotes their proliferation [20]. Moreover, butyrate regulates metabolism and maintains gut health by inhibiting histone deacetylase (HDAC) [21]. SCFA regulates the energy metabolism [22,23] and immunity, which generally depends on the G-protein-coupled receptors GPR43 (also known as FFA2), GPR41 (FFA3) [24] and GPR109A.

Besides dietary fiber, age is also an important factor shaping gut microbiota. For example, Bifidobacterium predominates in infants [25], while Bacteroides is more common in the elderly [26]. The gut microbiota of pigs has a succession rule [27]. The microbial diversity of pigs was significantly different with age, and this was mainly caused by diet, specially the neutral detergent fiber (NDF) in corn [28]. Surprisingly, it even can rapidly and reproducibly change the microbial community structure and gene expression in a day [29]. However, some studies have shown that neither eating a long-term vegan diet [30] nor a short-term high-fiber-low-fat diet significantly increase microbial diversity or SCFA [31]. The long-term dietary habit is the dominant force in establishing the composition of gut microbiota $[32,33]$, and gut microbiota might spend one year adapting to the dietary fiber in diet [34]. Therefore, the changes in the composition and quantity of the diet (e.g., a high-fiber or low-fiber diet) lead to short-term changes in the microbiota, while a long-term stable diet will not lead to a big change [35]. A longitudinal observation of the effects of diet on animals is needed [36], however, few such works have been made in chickens. In addition, studies have also shown that the same food produced different effects on gut microbiota in diverse people, which are highly individualized [37], and individual diets are currently under investigation. We speculated that this may be related to feed intake, the size of the cecum or colon and the amount of chyme.

Given this, we successively fed low-fiber, high-fiber and low-fiber diets to ISA Brown Hens $(\mathrm{IBH})$ and Chinese native breed Bian Hens $(\mathrm{BH})$ from during different growth periods $(1-8,9-20$ and 21-50 weeks), aiming to longitudinally observe the effects of altered dietary fiber on the gut microbiota, microbial metabolite SCFAs and the development of the cecum of chickens, and aiming to explore whether these are related to dietary fiber, feed intake, gut microbiota diversity and the seize of the cecum, as well as observing whether the trend is the same or not in two breeds of chickens.

\section{Materials and Methods}

\subsection{Animals and Experimental Design}

This experiment was approved by the Shanxi Agricultural University Animal Experiment Ethics Committee, and the license number was SXAU-EAW-2017-002Chi.001. In total, 108 one-day-old ISA Brown Hens(IBH) and 108 one-day-old Bian Hens (BH) with a 40 g average weight were chosen. IBH is a commercial breed while $\mathrm{BH}$ is a Chinese native breed with a character of crude fiber-tolerance. Each breed of chickens was randomly divided into 18 replicates of six chickens per 
cage. Chickens were fed three kinds of feed (Jinzhong Shiyang Feed Ltd, Taigu) (Table 1) containing different levels of dietary fiber (low-fiber, high-fiber and low-fiber) during 1-8, 9-20 and 21-50 weeks, respectively. Samples were harvested to measure the composition and function of gut microbiota, the concentration of SCFAs in the content of the cecum and the length of the cecum of $\mathrm{IBH}$ at the end of 8,20 and 50 weeks and $\mathrm{BH}$ at the end of 20 and 50 weeks.

Table 1. Ingredients and composition of feed used during different growth periods.

\begin{tabular}{|c|c|c|c|}
\hline Ingredients (\%) & $1-8$ & $9-20$ & $21-50$ \\
\hline Corn & 61.95 & 60.49 & 60.00 \\
\hline Soybean meal & 23.7 & 10 & 15.5 \\
\hline Bran & - & 8.5 & - \\
\hline Soybean oil & 1.1 & 0.5 & 0.6 \\
\hline Corn gluten meal & 4 & - & 1.6 \\
\hline Spray corn husk & - & 6.5 & 3.5 \\
\hline DDGS & 4 & 5.75 & 5 \\
\hline Peanut meal & - & - & 1 \\
\hline Stone power & 1.8 & 2.1 & 9.02 \\
\hline $\mathrm{CaHPO} 4$ & 1.3 & 0.7 & 0.65 \\
\hline $\mathrm{NaCI}$ & 0.3 & 0.28 & 0.25 \\
\hline Met & 0.2 & 0.06 & 0.14 \\
\hline Lys & 0.46 & 0.08 & 0.19 \\
\hline Thr & 0.09 & 0.04 & 0.09 \\
\hline Multivitamin & 0.4 & 0.35 & 0.4 \\
\hline Minerals & 0.55 & 0.5 & 0.45 \\
\hline Zeolite & - & 2 & 0.5 \\
\hline Choline chloride & 0.1 & 0.05 & 0.1 \\
\hline Complex enzyme & 0.05 & - & - \\
\hline Monosodium glutamate & - & 2 & \\
\hline Protein powder & - & 0.1 & 1 \\
\hline Total & 100 & 100 & 100 \\
\hline \multicolumn{4}{|l|}{ Feed composition (\%) } \\
\hline ME (MJ/kg) & 12.43 & 11.40 & 10.62 \\
\hline Crude protein & 19.49 & 15.3 & 16 \\
\hline Crude fiber & 3.21 & 3.95 & 2.95 \\
\hline Crude fat & 4.27 & 3.99 & 3.72 \\
\hline Crude ash & 5.83 & 5.67 & 12.13 \\
\hline $\mathrm{Ca}$ & 1.05 & 0.99 & 3.55 \\
\hline Total P & 0.57 & 0.5 & 0.43 \\
\hline $\mathrm{NaCI}$ & 0.3 & 0.37 & 0.31 \\
\hline
\end{tabular}

\subsection{Management}

Chickens were caged in brood cages for the first 1-8 weeks. After sampling at the end of the 8-week, chickens were transferred into three-step cages and raised until the experiment was completed. Chickens were given free access to water and feed. The management of the temperature, light, and humidity was conducted according to the breeding manual of IBH. No conventional immunization schedule of chickens was performed to avoid impacts on gut microbiota. Chicken manure was cleaned in a timely manner. Feed intake of each reduplicate of chickens was recorded.

\subsection{Sampling}

We chose nine chickens from nine replicates in IBH in 8 weeks, and chose nine chickens from each breed of chickens at the end of 20 and 50 weeks. They were executed with humanitarian 
slaughter and the length and weight of the cecum were measured. The contents of the right cecum were collected into multiple cryogenic tubes, and they were put into a liquid nitrogen tank; then preserved at $-80^{\circ} \mathrm{C}$ until the determination of SCFA. As above, the left cecum contents were gathered to perform the 16S rRNA gene sequence of gut microbiota.

\subsection{Determination}

\subsubsection{S rRNA Gene Sequence}

The $16 \mathrm{~S}$ rRNA gene of gut microbiota was sequenced by Genedenovo Biotechnology Ltd (Guangzhou, China) using High-Throughput Sequencing Technology. First, DNA extraction and PCR amplification were performed using the HiPure Stool DNA Kits (Magen, Guangzhou, China). V3-V4 regions of the16S ribosomal RNA gene were amplified by PCR using primers 341F 5'-CCTACGGGNGGCWGCAG and 806R 3'-GGACTACHVGGGTATCTAAT. Illumina Hiseq 2500 sequencing was successively extracted.

Bioinformatics analysis. (1) Quality control and reads assembly. (2) OTU cluster. Effective tags were clustered into operational taxonomic units (OTUs) of $\geqslant 97 \%$ similarity using the UPARSE pipeline[38]. The tag sequence with highest abundance was selected as representative sequence within each cluster. (3) Taxonomy classification. The representative sequences were classified into organisms by a naive Bayesian model using RDP classifier (version 2.2) [39] based on SILVA Database [40], with the confidence threshold values ranged from 0.8 to 1 . Biomarker features in each group were screened by Metastats (version 20090414) [41] and linear discriminant analysis effect size (LEfSe) software (version 1.0) [42]. (4) alpha diversity and beta diversity analysis. Alpha diversity indices including ACE, Chao1, Shannon and Simpson were calculated in QIIME. Beta diversity was performed using Muscle (version 3.8.31) [43]. (5) Function prediction. KEGG pathway analysis of the OTUs was inferred using Tax4Fun (version 1.0) [44].

\subsubsection{The Concentration of SCFAs}

It was measured using the internal standard method with High Performance Gas Chromatography (HPGC). First, we prepared a solution containing internal standard crotonic acid. We accurately weighed metaphosphoric acid $25 \mathrm{~g}$ and crotonic acid $0.6464 \mathrm{~g}$, and put them into a $100 \mathrm{~mL}$ volumetric flask and up to $100 \mathrm{~mL}$ with ultra-pure water. Then, we prepared $100 \mathrm{~mL}$ mixed standard stock solutions as follows: different volumes of standards were added (Table 2) into a 100 $\mathrm{ml}$ volumetric flask, topped up to $100 \mathrm{~mL}$ with ultra-pure water and preserved at $4^{\circ} \mathrm{C}$. The concentration $(\mathrm{g} / \mathrm{L})$ of additive was calculate according to the density of each standards (e.g., acetate is $1.050 \mathrm{~g} / \mathrm{ml}$ ) and then it was converted into the mol concentration $(\mathrm{mmol} / \mathrm{L})$ base on molar mass of each standard (e.g. acetate is $60 \mathrm{~mol} / \mathrm{g}$ ). The volatile fatty acid standard solution was prepared as follows: $0.2 \mathrm{~mL}$ of deproteinized metaphosphate solution containing crotonic acid was added to three $1.5 \mathrm{~mL}$ centrifuge tubes, and $1 \mathrm{~mL}$ mixed standard stock solution was added to this. The peak area of crotonic acid in the standard solution was measured.

Sample preparation: $0.5-1 \mathrm{~g}$ contents of the cecum were added to nine times the weight of ultra-pure water, homogenate, centrifuged at 10,000 rpm for $10 \mathrm{~min}$, and the supernatant was removed. Then, $1 \mathrm{~mL}$ of supernatant sample was placed into a $1.5 \mathrm{~mL}$ EP tube, and $0.2 \mathrm{~mL}$ mixed solution of crotonic metaphosphate was added, and reacted for $3 \mathrm{~h}$. Centrifugation at 12,000 r for 5 min was undertaken. The supernatant was injected into the chromatograph instantaneously with a $10 \mu \mathrm{L}$ microinjector, and the injection volume was $1.0 \mu \mathrm{L}$. Reaction conditions were set as follows: injection temperature $220^{\circ} \mathrm{C}$; initial temperature $70^{\circ} \mathrm{C}$; detector temperature $220^{\circ} \mathrm{C}$; split 5 ; split ratio 6; constant current $0.8 \mathrm{~mL} / \mathrm{min}$; tail blowing $40 \mathrm{~mL} / \mathrm{min}$; hydrogen $35 \mathrm{~mL} / \mathrm{min}$ and air $350 \mathrm{~mL} / \mathrm{min}$.

The concentration of a certain acid $(\mathrm{mmol} / \mathrm{L})=($ peak area of certain acid of sample $\times$ peak area of crotonic acid in standard solution $\times$ mol concentration of certain acid $) \div($ peak area of crotonic acid in sample $\times$ peak area of certain acid in standard solution). 
Table 2. The additive volume and concentrations of volatile fatty acid standards added to the standard stored solution.

\begin{tabular}{ccccccc}
\hline & Acetate & Propionate & Butyrate & Isobutyrate & Isovalerate & Valerate \\
\hline $\begin{array}{c}\text { Additive volume } \\
\mu \mathrm{L}\end{array}$ & 60 & 40 & 20 & 5 & 5 & 5 \\
$\begin{array}{c}\text { Concentration } \\
\text { g/L }\end{array}$ & 0.63 & 0.40 & 0.19 & 0.048 & 0.047 & 0.047 \\
$\begin{array}{c}\text { Mol concentration } \\
\text { mmol/L }\end{array}$ & 10.50 & 5.35 & 2.19 & 0.54 & 0.46 & 0.46 \\
\hline
\end{tabular}

${ }^{1}$ Concentration of additive standards $(\mathrm{g} / \mathrm{L})=$ density of standards $(\mathrm{g} / \mathrm{mL}) \times$ additive volume $(\mu \mathrm{L}) \div 100.2 \mathrm{Mol}$ concentration $(\mathrm{mmol} / \mathrm{L})=$ concentration of additive standard $(\mathrm{g} / \mathrm{L}) \div$ molar mass of standard $(\mathrm{g} / \mathrm{mol}) \times 1000$.

\subsection{Statistical Analysis}

Statistical analyses of SCFAs and the development of cecum were performed using a one-way analysis of variance (ANOVA) with Statistical Product and Service Solutions (SPSS) 22.0 (IBM). The results are expressed as the means and standard error of the mean (SEM). In terms of gut microbiota, abundance statistics of each taxonomy were visualized using Krona [45] (version 2.6). The comparison of $\alpha$-diversity indexes between groups was calculated by Welch's t-test and Wilcoxon rank test using Vegan package (version 2.5.3) in R project, and the comparison among groups was computed by Tukey's HSD test and the Kruskal-Wallis H test using Vegan package (version 2.5.3) in $\mathrm{R}$ project [46]. The $\beta$-diversity analyses of Welch's t-test, the Wilcoxon rank test, Tukey's HSD test and the Kruskal-Wallis H test were calculated using Vegan package (version 2.5.3) in R project.

\section{Results}

For simplicity, "ISA Brown Hens-eight weeks" was named IBHE, "ISA Brown Hens-twenty weeks" was named IBHT, and "ISA Brown Hens-fifty weeks" was named IBHF. Similarly, "Bian Hens-twenty weeks" was named BHT for short, and "Bian Hens-fifty weeks" was named BHF.

\subsection{Gut Microbial Composition, Function and Diversity During Different Growth Periods}

\subsubsection{The dominant bacteria during the different periods}

The top 10 dominant bacteria during the different periods were taxonomically classified (Figure, 1-3). A distinctive color histogram represents the relative distribution of these most dominant bacteria ( $>1 \%$ of the total sequences). The names of distinct levels of phyla, family and genus are shown on the right of figures and the names of samples are under the figure.

At the phylum level, Bacteroidetes (red) and Firmicutes (blue) were the main phyla in the two breeds of chickens during the entire experiment periods; however, the abundance of Bacteroidetes gradually increased while Firmicutes gradually decreased from 8 to 50 weeks. The dominant bacteria of IBHE and IBHT also included Proteobacteria (light blue) and Verrucomicrobia (beige), respectively.

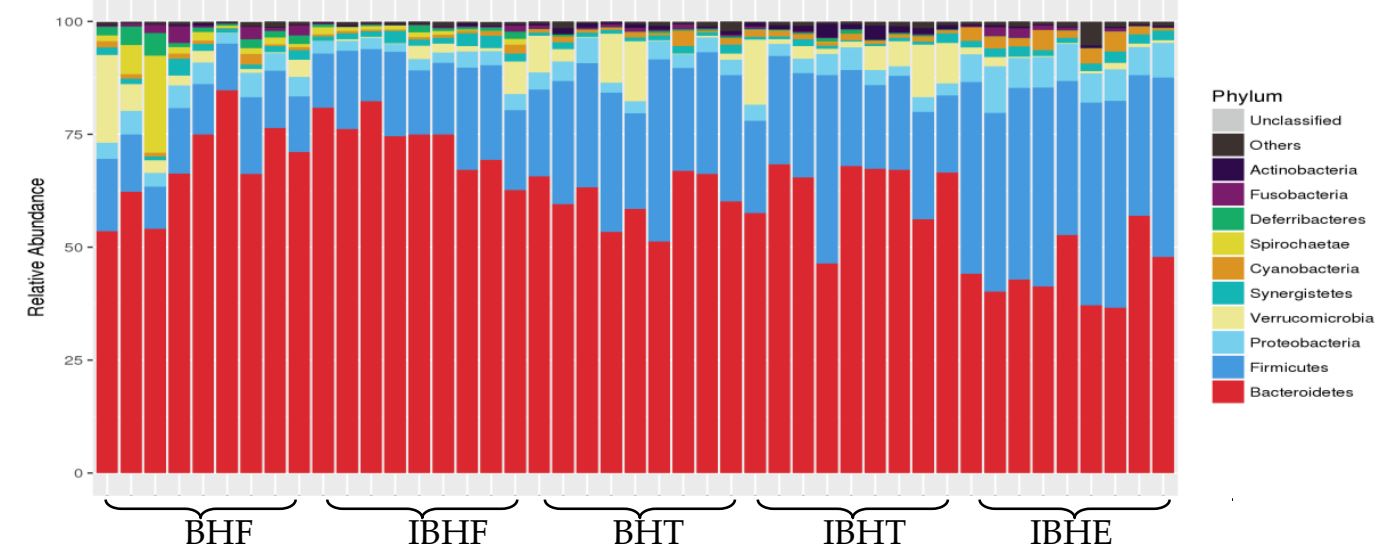


Figure 1. Taxonomy stack distribution of the top 10 dominant phyla in 8,20 and 50 weeks.

At the family level, the dominant bacteria in the two breeds of chickens was Bacteroidaceae (red), followed by Rikenellaceae and Ruminococcaceae. The dominant bacteria in 8 and 20 weeks included Lachnospiraceae which contains many butyrate-producing bacteria. The dominant bacteria at 20 and 50-week included Bacteroidales_S24-7_group (green) and fiber-degradation bacteria Prevotellaceae (blue green) and acetate-producing bacteria Acidaminoccaceae.

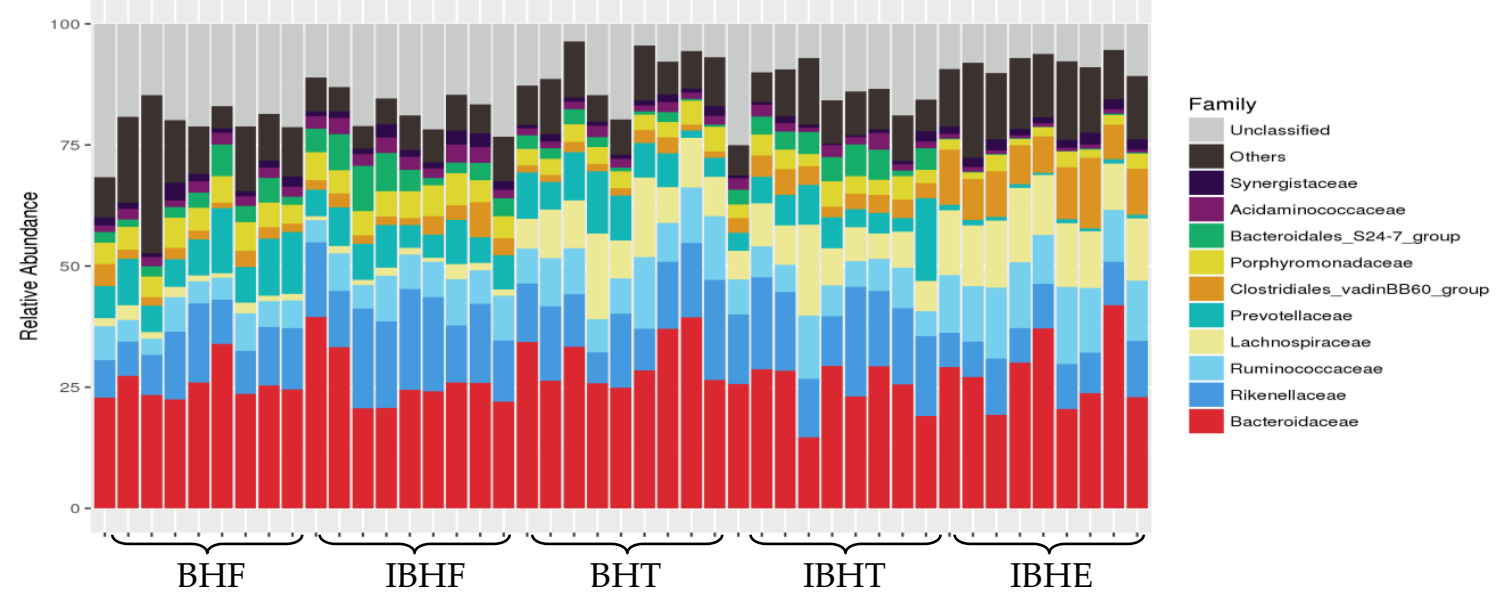

Figure 2. Taxonomy stack distribution of the dominant family in 8, 20 and 50 weeks.

We focused on the genus in this experiment. At the genus level, the dominant bacteria of 8-week-old chickens (IBHE) included the bile-tolerant bacteria Alistipes (beige) and torques_group (light blue). The dominant bacteria of 20-week-old chickens included the torques_group, Rikenellaceae_RC9_gut_group (dark blue), the potential fiber-degradation bacteria Prevotellae_UCG_001 (blue green) and Alloprevotella (green), Notably, the abundance of Alloprevotella is higher in BHT than IBHT, which suits to the character of the crude fiber-tolerant of BH. BHF and IBHF included Rikenellaceae_RC9_gut_group (dark blue), propionat-producing bacteria Phascolarctobacterium (brown) and Desulfovibrio (purple).

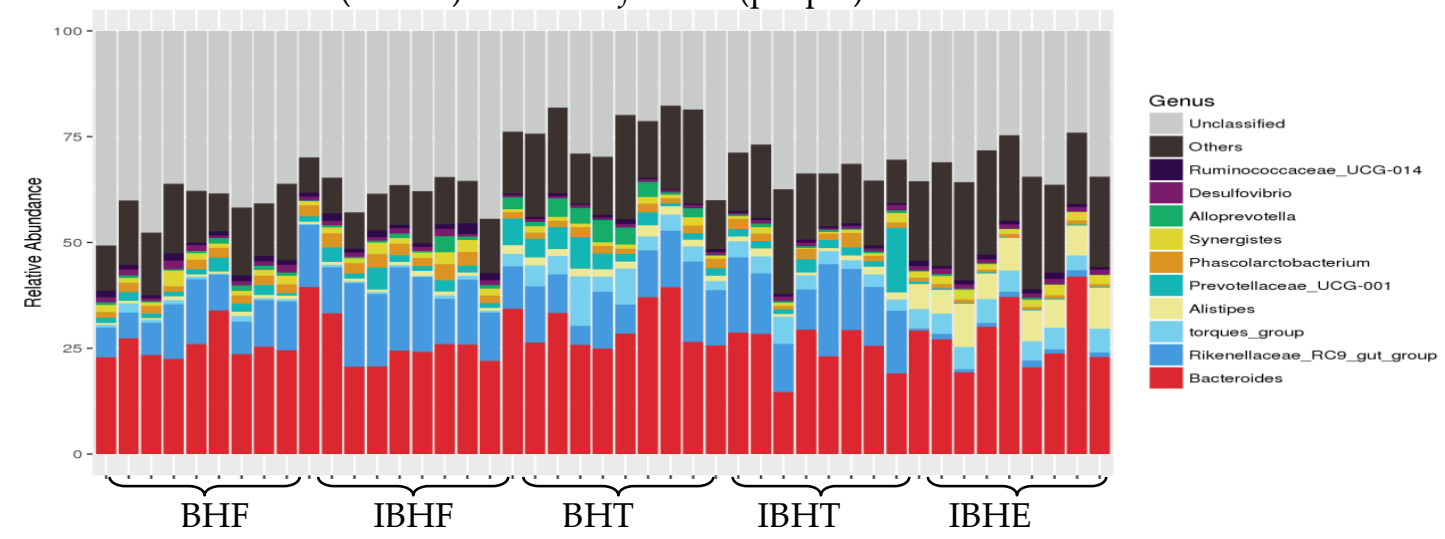

Figure 3. Taxonomy stack distribution of the dominant genera in 8, 20 and 50 weeks.

Furthermore, in order to better understand the effects of the fiber diet on genus, we observed the top 25 dominant bacteria genera through a heat map (Figure 4). This is consistent with Figure 3. The degree of heat gradually decreased with the color gradient turing blue from red.

This showed that the bacterial heat classification at 8, 20 and 50 weeks was distinct. We focused on fiber-degradation bacteria and SCFA-producing bacteria. The dominant bacteria of IBHE included fewer SCFA-producing bacteria and bile-tolerant bacteria Alistipes but no fiber-degradation bacteria, which was related to the low-fiber diet at 8 weeks. In contrast, IBHT and BHT with a high-fiber diet had more dominant SCFA-producing bacteria Phascolarctobacterium, Odoribacter, Faecalibacterium, Megamonas, Anaerotruncus and potential fiber-degradation bacteria 
Bacteroides, Prevotellaceae_UCG-001 and Alloprevotella. Notably, some fiber-degradation bacteria such as Bacteroides, Prevotella also produce SCFA. Notably, IBHF and BHF with the lowest-fiber diet also had some potential fiber-degradation bacteria such as Prevotellaceae_UCG-001, Alloprevotella and Prevotellaceae_Ga6A1_group and SCFA-producing bacteria Phascolarctobacterium, Odoribacter, Fusobacterium and Sutterella. This may be related to the high feed intake, which means a great deal of fiber was brought in at the same time.

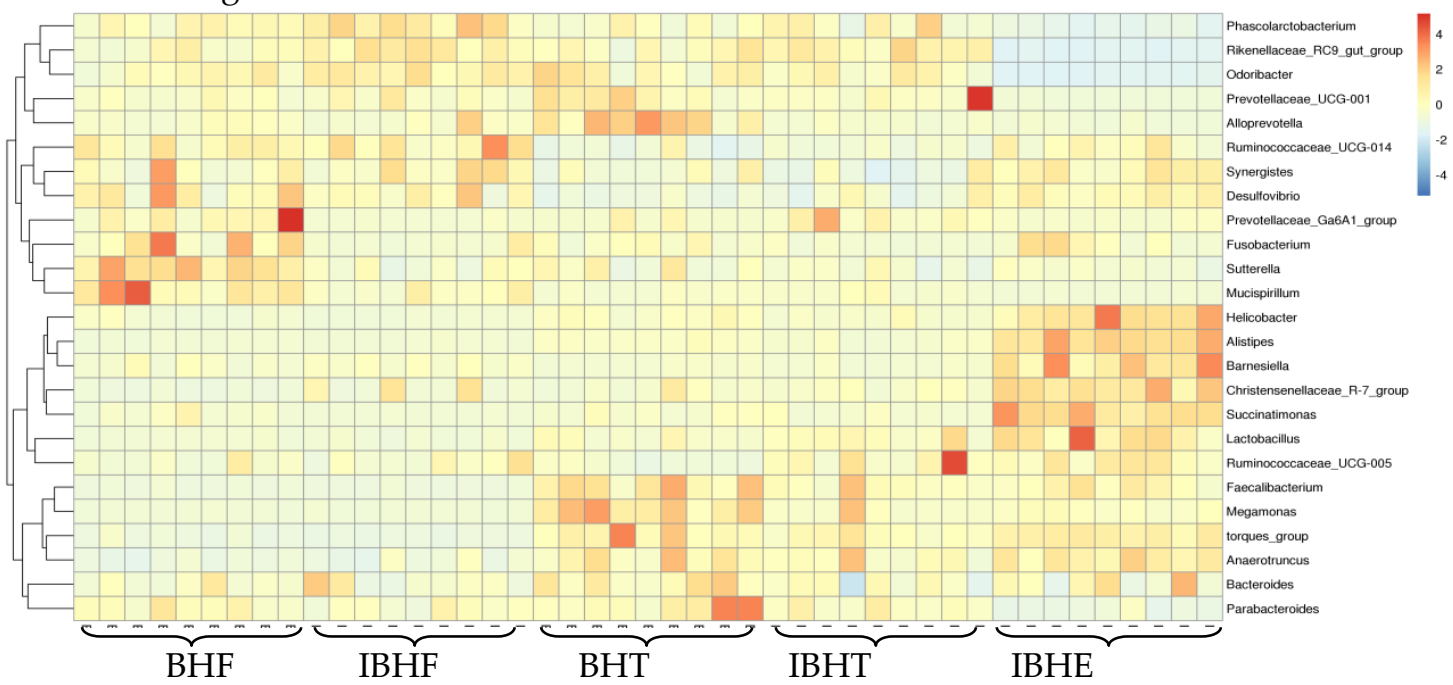

Figure 4. The heat map of dominant genera during different periods.

\subsubsection{Functional prediction of gut microbiota}

We further determined the functional prediction of gut microbiota further because many microorganisms have multiple functions. The heat map showed that the function annotation of microorganisms was significantly different during different growth periods (Figure 5). The functional annotation of 8-week bacteria were enriched in KEGG pathway K06147, K02003 and K01990, which all are defined by the ATP-binding cassette (ABC) transport system. This is because the dominant bacteria Firmicutes in 8 weeks introduces glucose via $\mathrm{ABC}$ transporters [14].

In contrast, functional annotation of 20 and 50-week bacteria not only included K06158, K15738 and K02004, which were defined by ABC transport, but also included K01190 ( $\beta$-galactosidase), K05349 ( $\beta$-glucosidase) and K00936 and K07636 which are defined by the two-component system, because the polysaccharide utilization site (PUL) of the dominant Bacteroidetes encodes a mixed two-component system (HTCs) and glycoside hydrolases [47, 48].

In addition, 50-week-old chickens also included K01897, which is defined as a long-chain acyl-CoA synthetase. K01897 includes the fatty acid metabolism k001212 and peroxisome proliferators-activated receptor (PPAR) signaling pathway ko03320, which is involved in the oxidation of fatty acids and glycogen storage in the liver. This was consistent with excess nutrition, overweight abdominal fat and fatty liver in 50-week-old chickens.

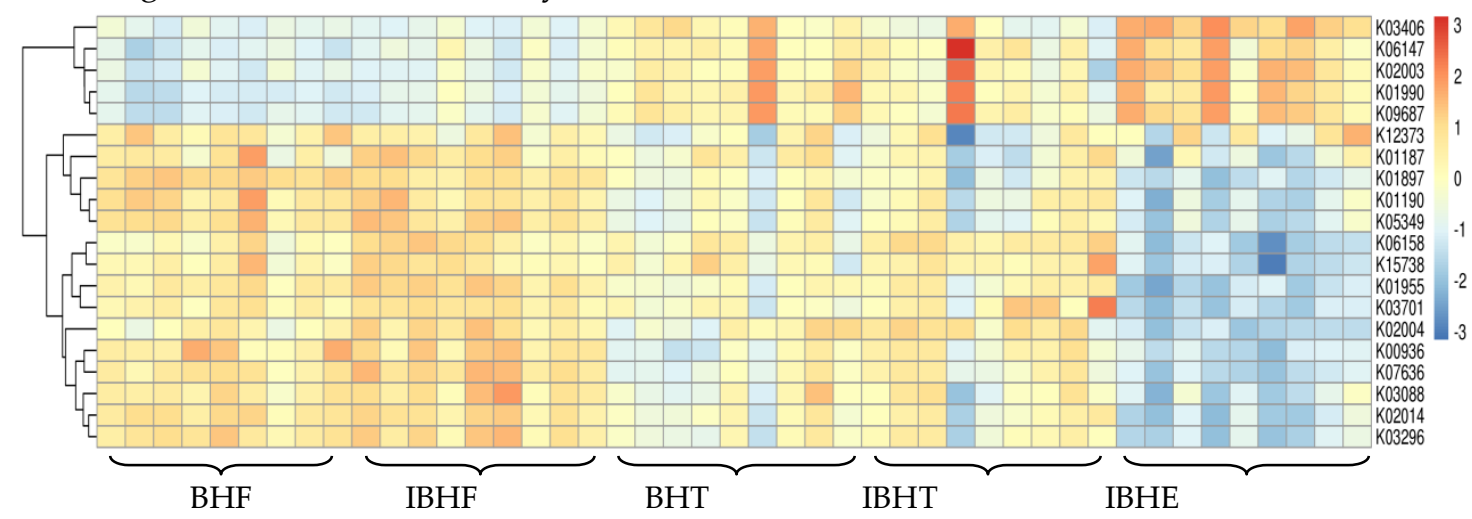


Figure 5. The heat map of functional prediction of OTUs during different periods.

\subsubsection{Gut microbial diversity indices of $\mathrm{IBH}$ and $\mathrm{BH}$}

Next, we measured gut microbial diversity during different growth periods. The $\alpha$-diversity reflects the microbial diversity within a single sample, while $\beta$-diversity is used to compare the microbial diversity between different samples. ACE and Chao1 reflect the community richness, while Shannon and Simpson indices reflect the community richness and community diversity.

It showed that the $\alpha$-diversity significantly increased with age except for Shannon and Simpson indices in IBH (Table 3). This was consistent with the numbers of OTUs which increased with age, especially during 20-50 weeks. The $\alpha$-diversity and $\beta$-diversity also significantly increased with age in BH (Table 3). Given the level of fiber was the lowest in 50 weeks, it suggested that the highest microbial diversity at 50 weeks might be induced by high feed intake but not fiber.

Table 3. Comparison of $\alpha$-diversity and $\beta$-diversity indices during different growth periods.

\begin{tabular}{|c|c|c|c|c|c|}
\hline & \multirow[b]{2}{*}{ Ace } & \multicolumn{3}{|c|}{ A-diversity } & \multirow[t]{2}{*}{ B-diversity } \\
\hline & & Chao1 & Shannon & Simpson & \\
\hline IBHE & $1561.90 \mathrm{Bc}$ & $1537.75 \mathrm{Bc}$ & 6.68 & 0.96 & 0.17 \\
\hline IBHT & $1782.80 \mathrm{Bb}$ & $1794.03 \mathrm{Bb}$ & 6.62 & 0.97 & 0.18 \\
\hline IBHF & $2370.16 \mathrm{Aa}$ & 2395.23 Аа & 6.91 & 0.97 & 0.22 \\
\hline SEM & 52.10 & 46.52 & 0.10 & 0.0050 & 0.018 \\
\hline $\mathrm{BHT}$ & 1761.79 в & 1783.53 в & $6.29 \mathrm{~b}$ & $0.96^{b}$ & $0.16^{\text {в }}$ \\
\hline $\mathrm{BHF}$ & $2174.98^{\mathrm{A}}$ & $2220.23 \mathrm{~A}$ & $6.96^{a}$ & $0.97^{a}$ & $0.29 \mathrm{~A}$ \\
\hline SEM & 41.83 & 40.33 & 0.083 & 0.0033 & 0.016 \\
\hline
\end{tabular}

Different superscript letters $(\mathrm{A}, \mathrm{B}$ ) and $(\mathrm{a}, \mathrm{b})$ represent an extremely significant difference and a significant difference in the same column respectively.

\subsection{Comparison of SCFA During Different Periods}

Next, we measured the relation between microbial diversity and SCFA (Table 4). Interestingly, although the microbial diversity at 50 weeks was the highest, the concentration of SCFA in IBHT was almost twice as high as in IBHE and IBHF. The concentration of propionate and butyrate in BHT was also significantly higher than those in BHF. Given that number and abundance of dominant SCFAs-producing bacteria in IBHT was similar to IBHF, it could not induce this big change; thus we speculated that there would be another primary reason.

Table 4. SCFAs (mmol/L) of IBH and BH during different growth periods.

\begin{tabular}{ccccccc}
\hline & Acetate & Propionate & Butyrate & Isobutyrate & Isovalerate & Valerate \\
\hline IBHE & $3.17^{\mathrm{Bb}}$ & $0.77^{\mathrm{B}}$ & $0.22^{\mathrm{B}}$ & $0.074^{\mathrm{B}}$ & $0.12^{\mathrm{B}}$ & $0.06^{\mathrm{B}}$ \\
$\mathrm{IBHT}$ & $5.84^{\mathrm{Aa}}$ & $1.92^{\mathrm{A}}$ & $0.59^{\mathrm{A}}$ & $0.22^{\mathrm{A}}$ & $0.30^{\mathrm{A}}$ & $0.17^{\mathrm{A}}$ \\
$\mathrm{IBHF}$ & $3.59 \mathrm{ABb}$ & $0.97^{\mathrm{B}}$ & $0.26^{\mathrm{B}}$ & $0.10^{\mathrm{B}}$ & $0.15^{\mathrm{B}}$ & $0.089^{\mathrm{B}}$ \\
SEM & 0.47 & 0.09 & 0.069 & 0.039 & 0.10 & 0.05 \\
BHT & 4.77 & $2.13^{\mathrm{a}}$ & $0.75^{\mathrm{a}}$ & 0.08 & 0.19 & 0.15 \\
BHF & 4.08 & $1.08^{\mathrm{b}}$ & $0.32^{\mathrm{b}}$ & 0.11 & 0.14 & 0.09 \\
SEM & 0.26 & 0.22 & 0.07 & 0.065 & 0.05 & 0.01 \\
\hline
\end{tabular}

Different superscript letters $(\mathrm{A}, \mathrm{B})$ and $(\mathrm{a}, \mathrm{b})$ separately represent an extremely significant and a significant difference in the same column.

\subsection{LEfSe of Significant Different Microbiota between 20 weeks and 50 weeks}

In order to discover the reasons that the SCFA values of IBHT were significantly higher than those in IBHF, we determined biomarkers genera using LEfSe (Figure 6). A value of Linear Discriminant Analysis (LDA) of certain microbes of $>2$ shows that the difference was significant. 
We focused on the number and abundance of fiber-degradation genus and SCFA-producing genera. This showed that compared with the IBHT, IBHF has more unique fiber-degradation bacteria with low-abundance, such as Prevotella_1, Treponema and Fibrobacter which was a specialist of degrading cellulose (Figure 6a). This was consistent with the comparison between BHT and BHF (Figure 6b). In addition, the number and relative abundance of dominant SCFA-producing bacteria (count fiber-degradation bacteria which can produce SCFA) showed little difference between IBHT and IBHF (Figure 6a) (Table 5). However, notably, the numbers of dominant genera and unique bacteria in 50-week-old chickens were found to be increased compared to 20 -week-old chickens using Metastats; however, the majority of them were not fiber-degradation or SCFA-producing bacteria (Table 6). This implies that so many bacteria competed for limited glucose that less glucose was fermented into SCFA by fewer SCFA-producing bacteria. This maybe a primary reason that the content of SCFA in IBHT was significantly higher than in IBHF.

Table 5. The numbers of significantly genera and unique genera in IBH and $\mathrm{BH}$.

\begin{tabular}{cccc}
\hline & Fiber-degradation genera & SCFA-producing genera & Unique genera \\
\hline IBHT & 4 & 14 & 16 \\
IBHF & 11 & 17 & 60 \\
BHT & 7 & 18 & 27 \\
BHF & 9 & 17 & 64 \\
\hline
\end{tabular}

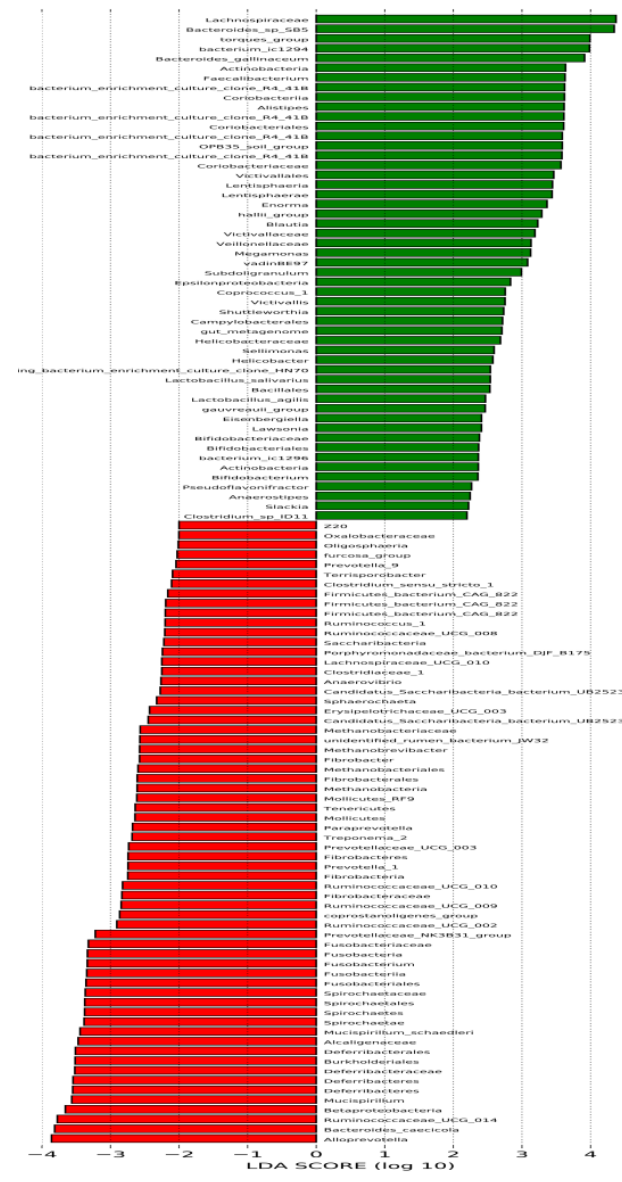

(a) IBHT VS IBHF

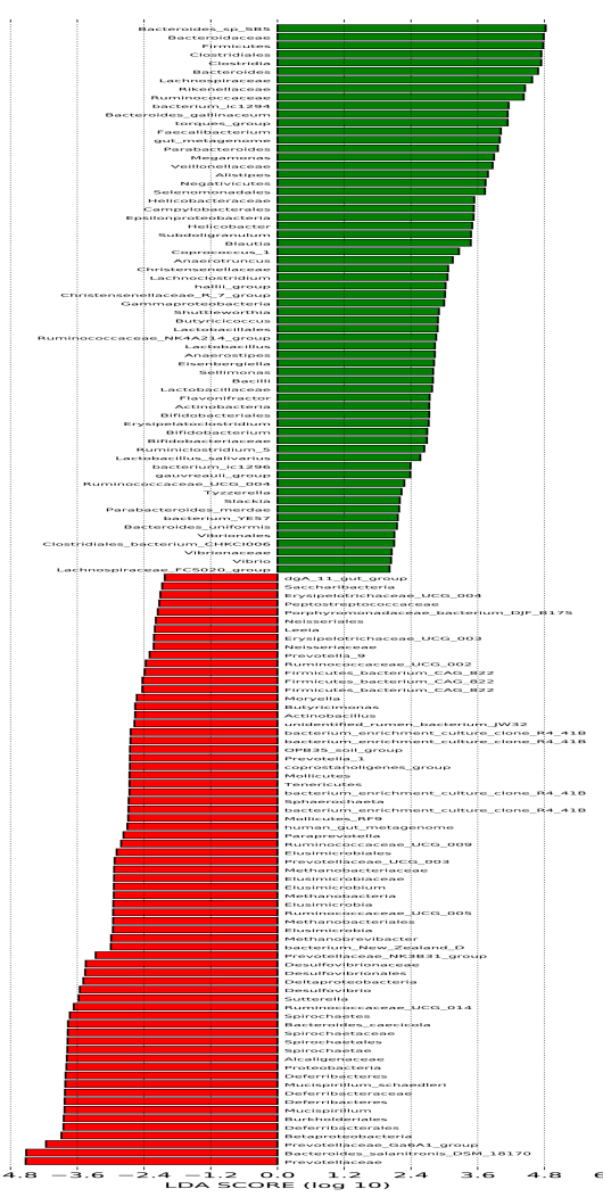

(b) BHT VS BHF

Figure 6. LDA of gut microbiota between IBHT and IBHF. 
In addition, we speculated that total SCFAs should be related to the size of the cecum. We found that the length of the cecum and the weight of the cecum chyme significantly increased from $8-20$ weeks in IBH (Table 6). This was possibly another reason that the SCFAs of IBHT were higher than IBHE. However, surprisingly, the length of the cecum shortened from 20-50 weeks both in $\mathrm{BH}$ and IBH (Table 6). It was uncertain whether the decreased cecum was a normal physiological phenomenon or induced by a low-fiber diet. In addition, it showed that the feed intake and weight of abdominal fat significantly increased with age, and fatty livers were also found in BHF and IBHF in this experiment.

Table 6. Comparison of feed intake and development of the cecum during different growth periods.

\begin{tabular}{ccccc}
\hline & $\begin{array}{c}\text { Feed } \\
\text { Intake }(\mathrm{g})\end{array}$ & $\begin{array}{c}\text { Weight of the abdominal } \\
\text { fat }(\mathrm{g})\end{array}$ & $\begin{array}{c}\text { Length of the } \\
\text { cecum }(\mathbf{c m})\end{array}$ & $\begin{array}{c}\text { Weight of the cecum } \\
\text { contents }(\mathbf{g})\end{array}$ \\
\hline IBHE & $51.53^{\mathrm{C}}$ & $5.72^{\mathrm{C}}$ & $12.14^{\mathrm{b}}$ & $0.90^{\mathrm{Bc}}$ \\
IBHT & $93.33^{\mathrm{B}}$ & $42.25^{\mathrm{B}}$ & $14.1^{\mathrm{a}}$ & $2.07^{\mathrm{Ab}}$ \\
IBHF & $126.18^{\mathrm{A}}$ & $85.77^{\mathrm{A}}$ & $12.95^{\mathrm{ab}}$ & $2.36^{\mathrm{Aa}}$ \\
SEM & $1.52^{\mathrm{B}}$ & $2.70^{\mathrm{B}}$ & 0.44 & 0.20 \\
BHT & $76.53^{\mathrm{B}}$ & $48.14^{\mathrm{B}}$ & 11.71 & 2.29 \\
BHF & $108.38^{\mathrm{A}}$ & $117.97^{\mathrm{A}}$ & 10.32 & 1.45 \\
SEM & 1.11 & $4.86^{2}$ & 0.24 & 0.14 \\
\hline
\end{tabular}

Different superscript letters (A, B, C) and (a, b, c) separately represent an extremely significant and a significant difference in the same column.

\section{Discussion}

\subsection{Dietary Fiber and Fiber-Degradation Bacteria}

The composition of gut microbiota of the same individual shifted during different periods $[28,49]$ due to the altered gut microbiota mainly being shaped by different diets, especially dietary fiber [50,51]. For example, Bifidobacteria, which is predominant in infants gradually decreases after babies eat solid food [25]. Prevotella gradually became the most diverse and predominant genus with the increase of dietary fiber and age in pigs [28].

In this experiment, the dominant bacteria of 8-week-old IBH did not include fiber-degradation bacteria but included Firmicutes, Proteobacteria and the bile-tolerant bacteria Alistipes. This was attributed to its high-fat-low-fiber diet [52]. Alistipes specially increased in the animal-based diet [29]. In contrast, the fiber-degradation bacteria Bacteroidetes, Prevotellae and Alloprevotella obviously increased in the high-fiber diet at 20 weeks and low-fiber diet at 50 weeks owing to the high feed intake. The abundance of Prevotellaceae was extremely low in mice fed a free-fiber diet [53]. Prevotella is enriched in a fiber diet [54] and has a strong ability to utilize fiber [55].

Notably, many unique fiber-degradation bacteria including Fibrobacter were found in a low-fiber diet at 50 weeks. This suggested that the increased feed intake of a low-fiber diet can also increase the abundance of fiber-degradation bacteria. Moreover, as far as we know, it seems that Fibrobacter has not been reported in chickens. Fibrobacter was once believed to only exist in mammalian intestines [56, 57]. Researchers first reported it in the cecum of birds (ostrich) in 2010 [58].

\subsection{Dietary Fiber and The Function Annotation of Bactreia}

The functional annotation of OUTs at 8 weeks included ABC transports. This was consistent with the dominant phylum, which was Firmicutes during this period. Contrary to the gram-negative bacteria Bacteroides, Firmicutes has gram-positive PULs (gpPULs) [14], which encode ABC transporters and the major facilitation superfamily (MFS) to introduce small sugar into the periplasm for processing. An ABC transporter is a type of transport ATPase on the bacterial plasma 
membrane and transfers glucose to the other side of the membrane through the change of conformation.

In contrast, functional annotation included two-component system and $\beta$-glycosidase in 20 and 50 weeks. Many members of Bacteroidetes can utilize polysaccharides through unique PULs [13]. PUL has been identified in all members of Bacteroides such as B. pleuroides and B. ovalis [59, 60]. PUL encodes a mixed two-component system (HTCs) and extracellular polysaccharide degrading enzymes such as beta-glycosidase [61,62]. Bacteroidetes can degrade dietary fiber by cleaving glycosidic bonds using extracellular glycoside hydrolase such as $\beta$-glucosidase [11]. The functional annotation at 50 weeks also included long-chain acyl-CoA synthetase, which contained fatty acid metabolism and PPAR signaling pathway. This was consistent with the increased abdominal fat and fatty liver of 50-week-old chickens. Acetate and butyrate can upregulate the target gene of PPAR $\alpha$, which can increase the oxidation of fatty acids and glycogen storage in the liver.

\subsection{Dietary fiber and SCFAs}

Dietary fiber is cleaved into monosaccharide by fiber-degradation bacteria before it is fermented into SCFA by SCFA-producing microorganisms [11]. SCFA is also called volatile fatty acid (VFA). Acetate, propionate and butyrate account for $90 \%$ 95\% of SCFA. Acetate-producing bacteria include Bifidobacterium, Sutterella and so on. Propionate-producing bacteria include Propionibacterium, Phascolarctobacterium, Veillonella [63] and so on. Butyrate-producing bacteria include Faecalibacterium[64], Roseburia, Coprocccus [65], Anaerostipes, Odoribacter and so on. SCFA-producing bacteria also contain fiber-degrading bacteria such as Bacteroides and Prevotella.

In this study, dominant SCFA-producing bacteria at 8 weeks were decreased, but included the bile-tolerant Alistipes and Bilophila. There was a significant positive correlation between Alistipes putradinis and the branched chain fatty acids (BCFAs) isobutyrate, and isovalerate [29]. About $16 \%-$ $-23 \%$ of the protein was fermented into BCFA with harmful metabolites such as ammonia. In contrast, 20-week IBHT and BHT included more dominant SCFA-producing bacteria. Therefore, SCFAs were increased compared to at 8 weeks. This was explained by the fact that the level of fiber increased in 20 weeks.

\subsection{Gut Microbial Diversity and SCFA}

The $\alpha$-diversity of the gut microbiota of pigs increases with age [28]. In this experiment, the microbial diversity also grew with age. Generally, high microbiota diversity and varied dietary fiber in the diet result in the increased production of multiple SCFAs [5]. Interestingly, we observed that the concentration of SCFAs (including BCFAs) in 20-week IBHT was greater than 50-week IBHF. We speculate that it is more likely that although the microbial diversity at 50 weeks was higher than that at 20 weeks, most of the bacteria were not fiber-degradation bacteria or SCFA-producing bacteria, this means that more bacteria compete for limited glucose and protein as the carbon and nitrogen sources for growth, and less glucose was fermented into SCFA including BCFAs. This may help to explain why obese people have less microbial diversity but have more SCFAs [66,67]. This is our new point of view. This also highlighted the importance of consuming dietary fiber that can increase the diversity of microorganisms including fiber-degradation bacteria or SCFA-producing bacteria and the content of SCFA such as acetate, propionate and butyrate, and decrease the fermentation of protein, the content of BCFAs and decrease the competition of other bacteria for monosaccharide.

\subsection{Dietary Fiber, Development of the Cecum and SCFAs}

Cecum is the principal place for microbial fermentation in chickens. We discovered that the length of the cecum in a high-fiber diet at 20 weeks was greater than that in a low-fiber diet at 8 weeks. Similarly, mice who were fed a high-fiber diet had a longer colon [4]. This supported the idea that the content of SCFA was increased at 20 weeks. In addition, surprisingly, the length of the cecum shortened in a low-fiber diet at 50 weeks. Because there are no related reports which describe this physiological phenomenon, it is hard to explain that whether it was just a physiological 
phenomenon or was caused by decreased dietary fiber or excessive nutrition at 50 weeks. The longer and thicker the cecum is, the more chyme would be accommodated, and more SCFAs and gases such as $\mathrm{CO}_{2}$ can be produced. Butyrate can provide energy for the proliferation of epithelial cells and development of the the cecum. Fermented gases expanded the volume of the cecum, thus in turn promoting more chyme to be accommodated, and more SCFAs can be produced. We discovered that the end of the cecum filled with gas in many chickens. This may be a different mechanism by which dietary fiber contributes to the production of SCFA.

Actually, the effects of SCFA on the health of the host are related to the total content of SCFAs in chyme. However, it seems impossible to weigh the total amount of chyme in the colon of humans or rumen of cattle. The size of the cecum (colon), total content of chime and SCFAs may partly explain that why the same diet produces different effects on the gut microbiota in different people [37].

\section{Conclusions}

The composition and function of gut microbiota, SCFAs and the size of the cecum are different during different growth periods, which is closely related to alerted dietary fiber in diets. The abundance of fiber-degradation and SCFA-producing bacteria is affected by feed intake besides dietary fiber. Similarly, the concentration of SCFA is not only affected by fiber-degradation bacteria and SCFA-producing bacteria, but also seriously affected by microbial diversity and the size of the cecum. The trend is the same in the two breeds of chickens. Notably, the length of the cecum decreased during 20-50 weeks; it is unclear whether this is just a normal physiological phenomenon or induced by reduced dietary fiber. In addition, the total amount of SCFA in the chyme should be investigated. Given that the causes for the same result were different during different growth periods in this experiment, long-term and longitudinal observations may be needed to better evaluate the effect of dietary fiber on chickens.

Author Contributions: conceptualization, Y.Y.; methodology, L.H.; formal analysis, B.S and L.H.; investigation, B.S. and L.H.; resources, Y.Y.; data curation, B.S. and L.H.; writing-original draft preparation, B.S.; writing - review and editing, B.S.; visualization, L.H.; supervision, Y.Y.; project administration, Y.Y. and B.S.; funding acquisition, Y.Y.

Funding: This research was funded by Key Research and Development Project Key Program of Shanxi Province (201703D211001); Technical System of Modern Agricultural Chicken Industry of Shanxi Province (20171102); Key Research and Development Project of Science and Technology (Agriculture) of Jinzhong City (201803D01100006); and Science and Technology Development Project Program of Shanxi Province (201603D221033-1). The APC was funded by Key Research and Development Project Key Program of Shanxi Province (201703D211001).

Conflicts of Interest: The authors declare no conflict of interest. The funders had no role in the design of the study; in the collection, analyses, or interpretation of data; in the writing of the manuscript, or in the decision to publish the results.

\section{References}

1. Stephanie L. Schnorr.; Marco Candela.; Simone Rampelli.; et al. Gut microbiome of the Hadza hunter-gatherers. Nature Communications, 2014, 5, 1-13.

2. Erica D. Sonnenburg.; Samuel A. Smits.; Mikhail Tikhonov.; et al. Diet-induced extinctions in the gut microbiota compound over generations. Nature. 2016, 529, 212-215.

3. Andrew H Moeller. The shrinking human gut microbiome. Current Opinion in Microbiology. 2017, 38, 30-35.

4. Mahesh S. Desai.; Anna M. Seekatz.; Nicole M. Koropatkin.; et al. A Dietary fiber-deprived gut microbiota degrades the colonic mucus barrier and enhances pathogen susceptibility. Cell. 2016, 167, 1339-1353.

5. Nishimura, S.; et al. CD8+ effector T cells contribute to macrophage recruitment and adipose tissue inflammation in obesity. Nature Med. 2009, 15, 914-920.

6. S Rahat-Rozenbloom.; J Fernandes.; GB Gloor.; et al. Evidence for greater production of colonic short-chain fatty acids in overweight than lean humans. International Journal of Obesity. 2014, 38, 1525-1531. 
7. Turnbaugh, P.J.; Hamady, M.; Yatsunenko, T.; et al. A core gut microbiome in obese and lean twins. Nature. 2009 , 457, 480-484.

8. Ley RE.; Bäckhed F.; Turnbaugh P.; Lozupone CA.; Knight RD.; Gordon JI. Obesity alters gut microbial ecology. Proc Natl Acad Sci USA. 2005, 102, 11070-11075.

9. Ridaura V K.; Faith J J.; Rey F E.; et al. Gut microbiota from twins discordant for obesity modulate metabolism in mice. Science. 2013, 341, 1079-U49.

10. Ara Koh.; Filipe De Vadder.; Petia Kovatcheva-Datchary.; et al. From dietary fiber to host physiology, short-chain fatty acids as key bacterial metabolites. Cell. 2016, 165, 1332-1345.

11. Harry J. Flint.; Edward A. Bayer.; Marco T. Rincon.; et al. Polysaccharide utilization by gut bacteria, potential for new insights from genomic analysis. Nature Reviews Microbiology, 2008, 6, 121-131.

12. Martens E C.; Lowe E C.; Chiang H.; et al. Recognition and degradation of plant cell wall polysaccharides by two human gut symbionts. PLoS Biology. 2011, 9, e1001221.

13. Johan Larsbrink.; Theresa E. Rogers.; Glyn R. Hemsworth.; et al. A discrete genetic locus confers xyloglucan metabolism in select human gut Bacteroidetes. Nature. 2014, 506, 498-502.

14. Sheridan PO.; Martin JC.; Lawley TD.; et al. Polysaccharide utilization loci and nutritional specialization in a dominant group of butyrate-producing human colonic Firmicutes. Microb Genom. 2016, 2, e000043.

15. Miyazaki, K.; Miyamoto, H.; Mercer, D.K.; et al. Involvement of the multidomain regulatory protein XynR in positive control of xylanase gene expression in the ruminal anaerobe Prevotella bryantii B4. J. Bacteriol. 2003, 185, 2219-2226.

16. Gorvitovskaia, A.; Holmes, S.P.; Huse, S.M. Interpreting Prevotella and Bacteroides as biomarkers of diet and lifestyle. Microbiome. 2016, 4, 15.

17. Tingting Chen.; Wenmin Long.; Chenhong Zhang.; et al. Fiber-utilizing capacity varies in Prevotella- versus Bacteroides-dominated gut microbiota. scientific reports. 2017, 7, 1-7.

18. Lior Artzi.; Edward A. Bayer and Sarah Moraïs. Cellulosomes, bacterial nanomachines for dismantling plant polysaccharides. Nature Reviews Microbiology. 2016, 15, 1-13.

19. Donohoe, D.R.; Garge, N.; Zhang, X.; et al. The microbiome and butyrate regulate energy metabolism and autophagy in the mammalian colon. Cell Metab. 2011, 13, 517-526.

20. W.E. Roediger. Role of anaerobic bacteria in the metabolic welfare of the colonic mucosa in man. Gut. 1980, 21, 793-798.

21. Valentina Tremaroli \& Fredrik Bäckhed. Functional interactions between the gut microbiota and host metabolism. Nature. 2012, 489, 242-249.

22. Troy S.; Soty M.; Ribeiro L.; Laval L.; Migrenne S.; Fioramonti X.; et al. Intestinal gluconeogenesis is a key factor for early metabolic changes after gastric bypass but not after gastric lap-band in mice. Cell Metab. 2008, 8, 201-211.

23. Katie C. Coate.; Steven A. Kliewer.; David J. Mangelsdorf. SnapShot, Hormones of the Gastrointestinal Tract. Cell. 2014, 159, 1478-1478.el.

24. Brown AJ.; Goldsworthy SM.; Barnes AA.; et al. The orphan G protein-coupled receptors GPR41 and GPR43 are activated by propionate and other short chain carboxylic acids. J Biol Chem. 2003, 278, 11312-11319.

25. Yatsunenko T.; Rey FE.; Manary MJ.; et al. Human gut microbiome viewed across age and geography. Nature. 2012, 486, 222-227.

26. Claesson M J.; Cusack S.; O Sullivan O.; et al. Composition, variability, and temporal stability of the intestinal microbiota of the elderly. Proceedings of the National Academy of Sciences. 2011, 108, 4586-4591.

27. Kim H B, Isaacson R E. The pig gut microbial diversity, understanding the pig gut microbial ecology through the next generation high throughput sequencing. Veterinary Microbiology, 2015, 177, 242-251.

28. .XiaoFan Wang.; Tsungcheng Tsai.; F eilong Deng.; et al. Longitudinal investigation of the swine gut microbiome from birth to market reveals stage and growth performance associated bacteria. Microbiome. 2019, 7, 1-1.

29. Lawrence A. David.; Corinne F. Maurice.; Rachel N. Carmody.; et al. Diet rapidly and reproducibly alters the human gut microbiome. Nature. 2014, 505, 559-577.

30. Wu GD.; Chen J.; Hoffmann C.; et al. Linking long-term dietary patterns with gut microbial enterotypes. Science. 2011, 334, 105-108.

31. Wu, G. D. et al. Comparative metabolomics in vegans and omnivores reveal constraints on diet-dependent gut microbiota metabolite production. Gut. 2016, 65, 63-72. 
32. Muegge B D.; Kuczynski J.; Knights D.; et al. Diet drives convergence in gut microbiome functions across mammalian phylogeny and within humans. Science. 2011, 332, 970-974.

33. CS Byrne.; ES Chambers.; DJ Morrison.; et al. The role of short chain fatty acids in appetite regulation and energy homeostasis. International Journal of Obesity. 2015, 39, 1331-1338.

34. Niv Zmora.; Jotham Suez and Eran Elinav. You are what you eat, diet, health and the gut microbiota. Nature Reviews Gastroenterology \& Hepatology. 2019,16, 35-56.

35. Palmer, C. et al. Development of the human infant intestinal microbiota. PLoS Biol. 2007, 5, 1556-1573.

36. Knight R.; Vrbanac A.; Taylor B C.; et al. Best practices for analysing microbiomes. Nature Reviews Microbiology. 2018,16,410-422.

37. Abigail J. Johnson.; PajauVangay.; Gabriel A. Al-Ghalith.; et al. Daily sampling reveals personalized diet-microbiome associations in humans. Cell Host \& Microbe. 2019, 25, 789-802.

38. Edgar, Robert C. UPARSE, highly accurate OTU sequences from microbial amplicon reads. Nature methods 10.10. 2013, 996-998.

39. Wang, Qiong, et al. "Naive Bayesian classifier for rapid assignment of rRNA sequences into the new bacterial taxonomy." Applied and environmental microbiology 73.16. 2007, 5261-5267.

40. Pruesse.; Elmar.; et al. "SILVA, a comprehensive online resource for quality checked and aligned ribosomal RNA sequence data compatible with ARB." Nucleic acids research 35.21. 2007, 7188-7196.

41. White.; James Robert.; Niranjan Nagarajan and Mihai Pop. "Statistical methods for detecting differentially abundant features in clinical metagenomic samples." PLoS Comput Biol 5.4. 2009, e1000352.

42. Segata, Nicola, et al. "Metagenomic biomarker discovery and explanation." Genome biology 12.6. 2011, 1.

43. Edgar, R.C. MUSCLE, multiple sequence alignment with high accuracy and high throughput. Nucleic Acids Res. 2004, 32, 1792-1797.

44. Oksanen J.; Blanchet F G.; Kindt R.; et al. Vegan, community ecology package. R package version 1.17-4. http, //CRAN.R-project.org/package=vegan. Acessoem. 2010, 23.

45. Ondov, Brian D.; Nicholas H. Bergman.; Adam M. Phillippy. Interactive metagenomic visualization in a Web browser. BMC bioinformatics 12.1. 2011, 385.

46. Aßhauer.; Kathrin P.; et al. Tax4Fun, predicting functional profiles from metagenomic 16S rRNA data. Bioinformatics. 2015, 31, 2882-2884.

47. D'Elia, J. N. \& Salyers, A. A. Effect of regulatory protein levels on utilization of starch by Bacteroides thetaiotaomicron. J. Bacteriol. 1996, 178, 7180-7186.

48. Sonnenburg E D.; Sonnenburg J L.; Manchester J K.; et al. A hybrid two-component system protein of a prominent human gut symbiont couples glycan sensing in vivo to carbohydrate metabolism. Proceedings of the National Academy of Sciences of the United States of America. 2006, 103, 8834-8839.

49. Harry J. Flint.; Karen P. Scott.; Petra Louis and Sylvia H. Duncan. The role of the gut microbiota in nutrition and health. Nature Reviews Gastroenterology \& Hepatology. 2012, 9, 577-589.

50. Rachel N. Carmody.; Georg K. Gerber.; Jesus M. Luevano.; et al. Diet dominates host genotype in shaping the murine gut microbiota. Cell Host \& Microbe. 2015, 17, 72-84.

51. Wang J.; Linnenbrink M.; Künzel, Sven.; et al. Dietary history contributes to enterotype-like clustering and functional metagenomic content in the intestinal microbiome of wild mice.. Proceedings of the National Academy of Sciences of the United States of America, 2014, 111, 2703-10.

52. Zhang, C. et al. Structural resilience of the gut microbiota in adult mice under high-fat dietary perturbations. ISME J. 2012, 6, 1848-1857.

53. Laurence Macia.; Jian Tan.;Angelica T. Vieira.; et al. Metabolite-sensing receptors GPR43 and GPR109A facilitate dietary fibre-induced gut homeostasis through regulation of the inflammasome. Nature Communications. 2015, 6, 1-15.

54. Carlotta De Filippoa.; Duccio Cavalieria.; Monica Di Paolab.; et al. Impact of diet in shaping gut microbiota revealed by a comparative study in children from Europe and rural Africa. PNAS. 2010, 107, 14691-14696.

55. Gorvitovskaia, A.; Holmes, S.P.; Huse, S.M. Interpreting Prevotella and Bacteroides as biomarkers of diet and lifestyle. Microbiome. 2016, 4, 15.

56. Ruengsomwong S.; La-Ongkham O.; Jiang J.; et al. Microbial community of healthy thai vegetarians and non-vegetarians, their core gut microbiota, and pathogen risk. Journal of Microbiology and Biotechnology. 2016, 26, 17-23. 
57. McDonald, J.E.; de Menezes, A.B.; Allison, H.E.; McCarthy, A.J. Molecular biological detection and quantification of novel Fibrobacter populations in freshwater lakes. Appl. Environ. Microbiol. 2009, 75, 5148-5152.

58. Montgomery, L.; Flesher, B.; Stahl, D. Transfer of Bacteroides succinogenes (Hungate) to Fibrobacter gen. nov. as Fibrobacter succinogenes comb. nov. and description of Fibrobacter intestinalis sp. nov. Int. J. Syst. Bacteriol. 1988, 38, 430-435.

59. Martens E C.; Lowe E C.; Chiang H.; et al. Recognition and Degradation of Plant Cell Wall Polysaccharides by Two Human Gut Symbionts. PLoS Biology, 2011, 9, e1001221.

60. Terrapon N , Lombard V , Gilbert H J , et al. Automatic prediction of polysaccharide utilization loci in Bacteroidetes species. Bioinformatics. 2015, 31, 647-655.

61. D'Elia, J. N. \& Salyers, A. A. Effect of regulatory protein levels on utilization of starch by Bacteroides thetaiotaomicron. J. Bacteriol. 1996, 178, 7180-7186.

62. Sonnenburg E D.; Sonnenburg J L.; Manchester J K.; et al. A hybrid two-component system protein of a prominent human gut symbiont couples glycan sensing in vivo to carbohydrate metabolism. Proceedings of the National Academy of Sciences of the United States of America. 2006, 103, 8834-8839.

63. Hilpert, W.; Dimroth P. Conversion of the chemical energy of methylmalonyl-CoA decarboxylation into a Na+ gradient. Nature. 1984, 296, 584-585.

64. Duncan SH.; Hold GL.; Harmsen HJ.; Stewart CS.; Flint HJ. Growth requirements and fermentation products of Fusobacterium prausnitzii, and a pro-posal to reclassify it as Faecalibacterium prausnitzii gen. nov., comb. nov. Int J Syst Evol Microbiol. 2002, 52, 2141-2146.

65. Susan E. Pryde.; Sylvia H. Duncan.; Georgina L. Hold, et al. The microbiology of butyrate formation in the human colon. FEMS Microbiology Letters. 2002, 217, 133-139.

66. Cho, I. et al. Antibiotics in early life alter the murine colonic microbiome and adiposity. Nature. 2012, 488, 621-626.

67. Schwiertz, A. et al. Microbiota and SCFA in lean and overweight healthy subjects. Obesity. 2010, 18, 190-195 